\title{
THE SPONTANEOUS RECONNECTION OF FORCE FREE MAGNETIC FIELD
}

\author{
LI XING and Y.Q. HU \\ Dept. of Earth and Space Science, Univ. of Sci. \& Tech. of China, Hefei \\ 230026, Anhui, P.R. China
}

\begin{abstract}
Two dimensional, three components compressible MHD equations are solved with multistep implicit scheme. The spontaneous reconnection of current sheets with force free field background is simulated. In comparison with the case where the background is potential field, a great difference is found. Our conclusion is that the force free magnetic field can accelerate reconnection. It may have important scientific significance to solar physics, for force free field is widely present in solar atmosphere.
\end{abstract}

\section{INTRODUCTION}

Both solar observation and theoretical study show that the enormous energy of eruptive phenomena in the solar atmosphere comes from the strong magnetic field. Because no force can compare to Lorentz force, the magnetic field must be force-free $(\mathrm{Hu}, \mathrm{1987})$. The study of how force-free field's energy is released has a very important significance. On the other hand, as a major mechanism for releasing magnetic energy fast, magnetic reconnection is the focus of solar physicists (Parker, 1981). It is found the plasma-moving velocity is very small before a solar flare (Hu, 1990). Free energy of force-free field can be released spontaneously, triggered by a current sheet (Li, 1992).

\section{NUMERICAL MODEL}

We introduce the so-called magnetic flux function to represent the magnetic field. For 2-dimensional problems in the perpendicular coordinates $(x, y, z)$, with $z$ the negligible coordinate, this flux function $\psi(x, y)$ is related to the magnetic field by

$$
B=\nabla \times\left(\psi e_{z}\right)+B_{z} e_{z},
$$

so that the divergence-free condition is automatically satisfied. Then the governing equations for MHD flows can be expressed in the form considering the effects of resistance and Joule heating $(\mathrm{Li}, 1992)$. 
We use a multi-step implicit scheme (Hu, 1991) to solve the equations in $\mathrm{Li}(1992)$. The domain of solution is taken to be $0 \leq x \leq 10 \delta, 0 \leq y \leq 20 \delta$ and subdivided into a $21 \times 22$ mesh, 21 points in the $x$-direction and 22 in $y$ direction. The grid points are equally spaced in $y$-direction but have an increasing spacing in $x$-direction.

Initial equilibrium state of the current is

$$
B_{x}=0
$$

$$
\begin{aligned}
& \left\{\begin{array}{l}
B_{y}=B_{0} \sin (\alpha x) \sin \left(\frac{\pi}{2 \delta x}\right) \frac{x}{|x|}, \\
B_{z}=B_{0} \cos (\alpha x) \sin \left(\frac{\pi}{2 \delta x}\right),
\end{array} \text { when }|x|<\delta\right. \\
& \left\{\begin{array}{l}
B_{y}=B_{0} \sin (\alpha x), \\
B_{z}=B_{0} \cos (\alpha x),
\end{array} \text { when }|x| \geq \delta\right. \\
& \rho(x)=\rho_{0}\left(1+\frac{B_{0}^{2}}{2 \mu_{0} v_{0}^{2}} \cos ^{2}\left(\frac{\pi}{2 \delta x}\right)\right), \quad \text { when }|x|<\delta \\
& \rho(x)=\rho_{0}, \quad \text { when }|x| \geq \delta
\end{aligned}
$$

where $\alpha$ is force-free field factor, $\delta$ is thickness of current sheet.

The next step in the simulation is to consider the boundary condition. At $x=0, y=0$ we use symmetrical boundary condition. At $x=10 \delta$ we have

$$
\begin{aligned}
& \psi^{n+1}(1, y)=\psi^{n}(1, y)+\psi^{n+1}(2, y)-\psi^{n}(2, y), \quad B_{z}^{n+1}(1, y)=B_{z}^{n}(, y), \\
& v_{z}(1, y)=v_{y}(1, y)=0, v_{x}(1, y)=v_{x}(2, y), T(1, y)=T(2, y), \rho^{n+1}(1, y)=\rho^{n}(1, y) ;
\end{aligned}
$$

at $y=20 \delta$ we use

$$
\psi^{n+1}(x, 1)=\left\{\begin{array}{l}
\psi^{n}(x, 1) \text { if } v_{y}(x, 1)<0 \\
\psi^{n}(x, 1)-v_{y}(x, 1) \frac{\partial \psi}{\partial y} \text { if } v_{y} \geq 0
\end{array}\right.
$$

\section{NUMERICAL RESULTS}

Numerical results are shown in Figures 1-3. Two typical cases are studied.

Case 1 As a special case of a force-free field, a potential field is studied: the force-free field factor is zero. Figure 1a gives an explicit evolutionary graph 

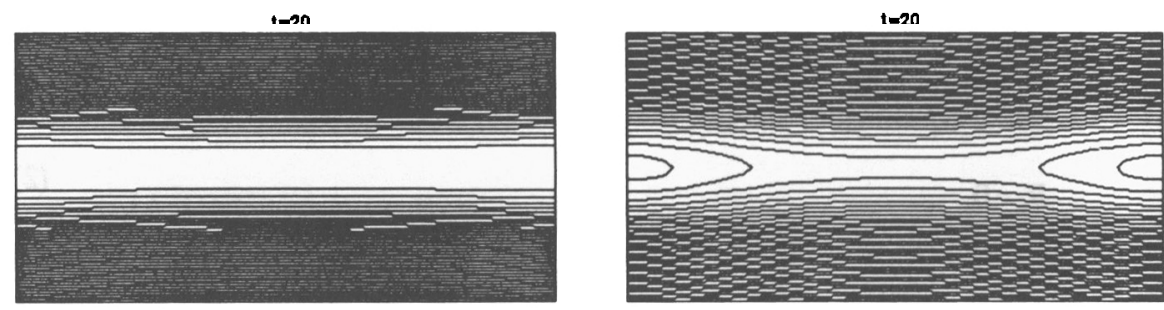

$t=40$

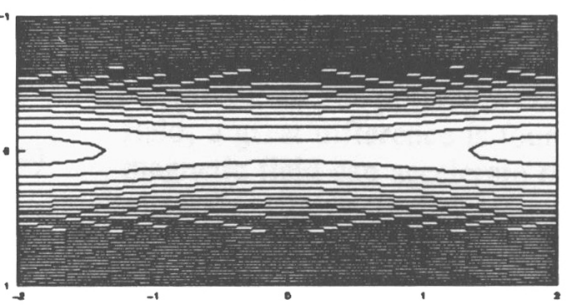

$t=40$

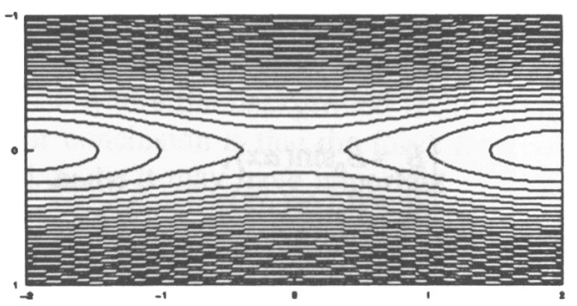

(a)

(b)

Fig. 1. Evolution of magnetic field line in (a) potential field background (b) force-free field background.

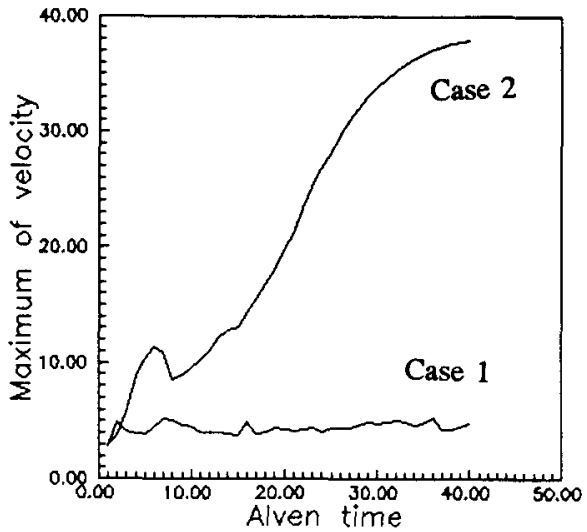

(a)

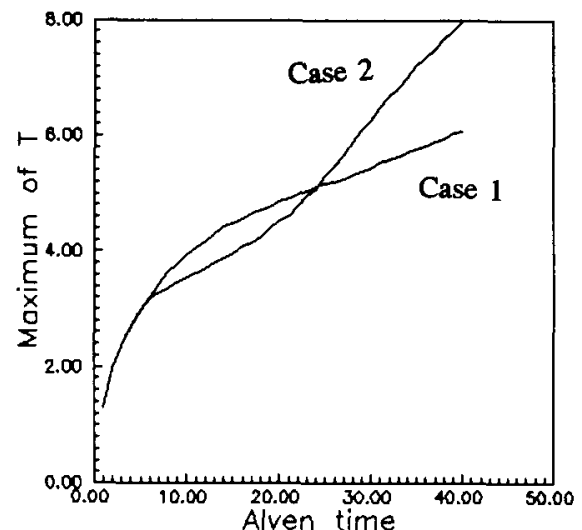

(b)

Fig. 2. Variation of (a) plasma's maximum velocity (b) plasma's highest temperature.

of magnetic field lines with time. It can be seen that the topological structure 
hardly changes when the background field of the current sheet is a potential field. Field lines go to the diffusion region in a column. Magnetic reconnection ratio is very small. So we conclude that a current sheet cannot bring about fast spontaneous reconnection when its background is a potential field.

Case 2 A quite different case is when the force-free field factor is nonzero. Figure $1 \mathrm{~b}$ shows the field lines' evolutionary diagram; no driven force is added. It is easy to see that not only magnetic merging but also reconnection appears in the diffusion region. At $t=10 t_{A}$, magnetic reconnection has happened, but the magnetic arch is very thin; at $t=20 t_{A}$, the magnetic arch's thickness has increased greatly. At $\mathbf{4 0} t_{A}$, the arch's thickness is much bigger than the initial thickness of the current sheets $\left(0.2 L_{0}\right)$.

The plasma's maximum velocity as a function of time is shown in Figure 2a. The plasma's velocity in the force-free field case is much greater than in the potential one. That is to say, more kinetic energy is released in Case 2. Figure 2b shows how the plasma's highest temperature changes with time. It is found that the temperature in Case 2 is higher also only if the time is long enough.

From the numerical results above, we conclude that in a current sheet with a force-free background, fast magnetic reconnection can happen spontaneously. This may be used to explain flares and other solar activities.

This research was supported by the Youth Foundation of University of Science and Technology of China \& Astronomy Committee Foundation of the Academy of Sciences of China.

\section{REFERENCES}

Hu, Wenrui, 1987, Cosmic Magnetohydrodynamics, Science Publishing House, p. 122.

Hu, Y.Q., 1990, in Proc. 7th Quadrennial STP Symposium ed. K.D. Cole et al., the Hague, Holland, p. 25.

Hu, Y.Q., 1991, J. Comput. Phys., 84, 441.

Li, X., Hu, Y.Q., 1992, Chinese Journal of Space Science, 12, 185.

Parker, E.R., 1981, Solar Flare Magnetohydrodynamics, ed. Priest, Gorden and Breach Sci. Publ., p. 139. 